\title{
21. Why gender-sensitive social protection is critical to the COVID-19 response in low- and middle-income countries
}

\author{
Melissa Hidrobo, Neha Kumar, Tia Palermo, Amber Peterman, \\ and Shalini Roy
}

Many governments are using social protection programs to respond to the economic crisis and health risk induced by COVID-19. As of April 17, 133 countries had adapted or introduced 564 social protection initiatives, according to the World Bank. With the focus on rapid assistance, gender considerations have understandably not been at the forefront of these efforts. A rapid assessment of initial COVID-19 social protection responses indicates that only $11 \%$ show some (albeit limited) gender-sensitivity.

This is unsurprising - most existing social protection programs in low- and middle-income countries (LMICs) are either gender-blind or neutral at best - but it is worrying. The COVID-19 crisis has the potential to widen gender inequalities, including those related to loss of livelihoods, reproductive health risks, disproportionate burden of care, and violence against women and children. Social protection that does not take gender into account can reinforce these inequalities.

General guidelines for COVID-19 social protection responses are available, but how can governments address gender inequalities? Designing gender-sensitive programming is not always straightforward, but evidence suggests simple design and implementation adaptations can make programming more gender-sensitive. While there is no one-size-fits-all approach, in a new brief summarized below, we provide key lessons, considerations, and guidance across five areas.

\section{Adapting existing schemes and choosing the forms of social protection}

Adapting existing schemes to be contagion-safe is a likely first step for governments, and these adaptations can have gender implications. Relaxing existing conditions (for example, those tied to work, health, or schooling) can simultaneously reduce viral spread and benefit women who are often responsible for fulfilling conditions, may be mobility-constrained, and may have fewer social or information networks. 
Expanding access to healthcare via fee waivers or providing automatic health insurance enrollment can support women in continuing to seek care for critical, routine maternal and child health and reproductive health services. Cash benefits (via e-payments) are widely recommended; cash can also improve household economic security and emotional well-being, which directly benefit women and can contribute to reducing intimate partner violence. However, the feasibility of safely providing additional in-kind transfers (including food or soap) should be considered as well, as women and children are often the first to reduce food consumption in response to food insecurity, and women may be responsible for daily shopping, exposing them to potential infection. In-kind transfers should be considered where mobility is restricted, markets are limited, food prices spike, or COVID-19 restrictions induce supply chain closures.

When social distancing restrictions are relaxed, implementers of public works programs should ensure dignified work with fair wages where women can safely participate, with exemptions for lactating and pregnant women. When schools reopen, implementers should pay particular attention to re-enrollment of adolescent girls and relax economic constraints with appropriate policy instruments.

\section{Targeting}

How to target households and individuals are critical considerations. Retaining the original individual-level targeting of many existing programs may be most straightforward; however, such targeting can exclude vulnerable populations. For example, unemployment insurance typically does not cover informal workers, including the majority of women who primarily work in the informal economy. Providing universal household-level transfers can reach more vulnerable people, but who the household's "named recipient" is may also have gender implications. Although broader evidence is mixed, a few studies from LMICs indicate that naming female recipients may improve women's empowerment. We believe the evidence supports considering women as named recipients - while recognizing that particularly acute periods of the crisis (such as lockdowns) may intensify household tensions.

Therefore, in settings where existing analysis shows the feasibility and acceptability of targeting women, we see gains in continuing during the COVID-19 crisis. But in settings where targeting women was previously deemed infeasible, we do not recommend starting during the crisis and explicitly challenging norms during a time when tensions are high. Nonetheless, even in the latter case, minor tweaks in operationalizing targeting - including authorizing multiple household members to make transactions, ensuring information reaches both men and women, and providing messaging that benefits are for the entire family - could contribute to greater gender equity.

\section{Benefit levels and frequency}

Benefits in response to COVID-19 should be quick and lumpy, ensuring sufficient support before supply chains are overwhelmed - and to avoid health risks from more frequent payment distributions and contact. While qualitative studies indicate that women may be able to retain control of smaller transfers, large randomized studies suggest larger cash transfer values result in higher benefits for households and women specifically. In addition, no studies we are aware of show that larger transfers to women induce adverse effects. 
Therefore, we believe programs should provide sufficiently high benefit levels to cover the duration of the COVID-19 economic crisis, understanding that programming during this time may be a full income replacement, rather than supplement. In addition, "top ups" should be considered for households caring for sick members or children to address disproportionate care burdens. Finally, it is important to consider that female-headed households are often smaller - and thus may appear better-off in a direct per capita poverty measure - yet may still be more disadvantaged for numerous reasons (for example, discrimination or access to services).

\section{Delivery mechanisms and operation features}

Programs generally employ the most logistically feasible delivery mechanisms and operational features in crisis conditions, but seemingly simple choices may have gender implications. Accessible grievance mechanisms should be set up, and implementation and management staffing should include women. Delivery mechanisms for benefits and information should be practical and accessible to both men and women.

While e-payments may not be an option for many settings, in the longer term, national programs should invest in these. Extending the network of e-payments may increase financial inclusion, including among women, who have lower inclusion rates. Responses should consider that in many settings women are less likely to have access to mobile phones; existing programs have sometimes provided them for this reason. While mobile phones are a promising platform for providing information, it is important to keep in mind that improving access alone may not be sufficient; women also have lower literacy, lower ability to pay for services, and multiple constraints on their time. Thus, mobile phonebased platforms should be complemented by other platforms such as internet, television, and radio; and when possible in mobile platforms, voice messages or speaking directly to an expert are preferred to text messaging. Women's groups or other peer support groups may be leveraged as networks for more efficient communication and delivery of essential services.

\section{Complementary programming}

Complementary programming remains relevant for women during COVID-19, especially on topics related to food and nutrition, including ways to access or grow nutritious foods when markets and supply chains are down; water and sanitation, as information about hygiene and social distancing is critical for reducing COVID-19 spread; maternal health including antenatal care, as travel may be restricted and health centers overburdened and a potential infection risk; sexual and reproductive health, including family planning and menstrual hygiene management; parenting and learning for children as many schools are closed; mental health for both men and women, given that many may experience depression related to isolation or loss of livelihoods; and access to referrals for violencerelated services.

All of these comprehensive services will rarely be available, particularly during the pandemic, but social protection platforms can at a minimum explore integrating light-touch information campaigns with delivery taking into account the gender considerations outlined above and linkages to services. 


\section{Concluding thoughts}

The COVID-19 pandemic presents an opportunity to address existing gender inequalities through social protection. Program designs should be adjusted to account for gender, in a manner informed by existing analysis, while taking a long-term approach. Related issues of political economy, coordination, and financing that have gender considerations should be explored in future guidance. Because these are complex issues and unintended consequences of programming are possible, more research is needed on intersections of social protection, gender, and pandemics, where ethically feasible. At a minimum, monitoring statistics should be sex- and age-disaggregated and, where possible, data should be collected to ensure risks to beneficiaries do not increase. Taken together, these policy adjustments and new evidence can lay the groundwork for more gender-sensitive social protection systems in LMICs both during the crisis and beyond.

This work was undertaken in collaboration with the Transfer Project and as part of the CGIAR Research Program on Policies, Institutions, and Markets (PIM) led by IFPRI.

Originally published April 28, 2020. 